\title{
Sentence Structure Balance in English Translation of Chinese
}

\section{Zhuosheng Yang}

School of Foreign Languages, Neijiang Normal University, Neijiang Sichuan, 641199, Chna

Keywords: The end-weight principle, Structure balance, English translation of Chinese

\begin{abstract}
In translation practice, structurally imbalanced sentences written by students are often seen; especially long sentences lack beauty, seriously affecting the quality of the translation. This paper explains the basic principles English sentence structure balance follow, deeply discusses structure imbalance problem of English sentence and appropriate treatment strategies, to be conducive for translation practice.
\end{abstract}

\section{Introduction}

In daily teaching or English Band 4, 6, and 8 paper scoring, English teachers often see the common phenomenon that there seems no problem in sentences translated by English learners, and the expression is faithful and fluent, but we feel the quality is not high, not on the grade and we even suspect English level of translators. This is deplorable. The reason, in fact, is that sentences lack structural beauty, and have structure imbalance problem.

English structure is governed by the two rules of end-focus and end-weight, while Chinese one only by the rule of end-focus. When translating, a translator can not copy the information structure of the source language into the target language. (Chao Y. R. 1969; Tang Tingchi, 1985; Quirk, 1985; etc).

As can be seen from the above, the order of English sentences is constrained by two principles, one of which is The Principle of End-Weight, the other of which is The Principle of End- Focus. This paper focuses on the sentence structure balance problem, and the principle of end-focus is excluded.

\section{The principle of end-weight}

In the process of constructing English sentences, we often hear "end-heavy", "end-core" or "the principle of end-weight". In fact, they are the principle of end-weight. Look at the statement of the linguist Leech, etc.:

Leech and Svartvik said that in order to keep the sentence structure balance and conform to the conventional expression form, people will put the "heavy" part in the end of the sentence; or the sentence will look clumsy or not balanced. The "heavy" part will be different in various situations in which preposition phrases are heavier than nouns; phrases including many words are heavier than phrases including a few words; complicated sentence structures are heavier than simple sentence structures, which are generally called "end-weight”. (Leech, G. \& Svartvik,J. A. A Communicative Grammar of English. London: Longmans, 1975)

According to the statement of Leech and other linguists, in order to maintain sentence structure balanced and follow traditional forms of expression, people often put "heavier" part on the end of the sentence, otherwise sentence will appear heavy and unbalanced. As for what is the "heavy" part, Leech says, in different circumstances, "heavy" is also different. Generally speaking, the prepositional phrase is "heavier" than noun, phrase with more words is "heavier" than one with fewer words, and complicated sentence structure is "heavier" than simple sentence structure. "Heavy" part is placed on the end, which is commonly referred to as the end-weight principle. Thus, relatively long or core sentence element should be placed at the end of the whole sentence, making the overall as positive trend triangle in order to achieve balanced and stable sentence pattern. This principle is quite widespread not only at the sentence level, but also at the phrase level.

Phase end-weight. From the microscopic point of view, the end-weight principle also appears in the phrase construction. The post-attributive in English follows this principle. Various types of 
phrases (including prepositional phrase / participial phrase / infinitive phrases, etc.) as well as attributive clause become typical post-attributive:

\section{A Puma at Large 逃遁的美洲狮 \\ a woman picking blackberries 正采摘黑莓的妇女 \\ a very agreeable situation located within two small hills 居于两山间的好位置 \\ Pumas are large, cat-like animals which are found in America. 见于美洲的猫科动物}

Above underlined word phrases are placed behind the central word, equivalent to an adjective, modifying the previous central word, called post-attributive in English, and all follow the principle of end-weight. While in Chinese, all are translated into pre-attributives. The whole phrase is a prejudiced phrase with modifier-core structure, totally not bound by the constraints of this principle.

Sentence end-weight. Macroscopically speaking, English sentence also has the tendency to put long, complex constituents at the end of sentences, which means the principle of placing the long and complex parts at the end of sentence in, so that the sentence structure can maintain balanced and symmetry. Please see:

1). He studied them that night with the kind of care his wife had suggested.(CGEL)

2). John offered Mary the help she needed.(CGEL)

3). I gave a book to each of the boys.(ALD)

4). A number of cars are already on the road powered by gas turbines.

5). It's certain that we'll forget the address.

6). When a thief was caught on the premise of a large jewellery store one morning, the shop assistant must have found it impossible to resist the temptation to say 'it serves him right'.

Underlined parts in above sentences are relatively long and heavy, placed at the end of sentences, so that the sentences look more beautiful and symmetry, which is the form beauty of sentence.

\section{Easily imbalanced structures in English sentence}

Among basic sentence patterns in English, SVC and SV, these two patterns are more prone to be imbalanced because these two sentence patterns do not have any object. Since the object is acted by noun or pronoun, vulnerable to be limited by modification, so more sentence parts are more easily added. If object is missing, there is not much composition to modify the definitive and the sentence is likely to be ended, that is to say, the end of the sentence is light, and seems thin. The subject is also acted by noun or pronoun, most likely to add modified definitive composition, so there will be end-light sentences, and end-weight principle is affected and the sentence structure balance will be threatened. Please see:

7). To think that a dangerous wild animal is still at large in the quiet countryside is disturbing.

8). Those who are contented are happy.

9). The time to explore the South Pole has come.

Of course, in SVC structure, C may also be acted by noun or pronoun. Here, C is specifically acted by the adjective.

Another case is likely to appear in SVO, SVoO, SVOC sentence patterns. In these sentences, $\mathrm{O}$ is used as the subject of the sentence to form passive sentences. These sentences are also prone to structurally imbalanced.

10). The story of her phenomenal success in Australia is told.

11). Decisions to install safety devices on all machines have been made.

After reading above sentence 7 to 11, do you feel they are familiar? Is there any problem? Please see the following specific analysis and explanation about how to achieve end-weight principle in translation, and get translated sentences with form beauty.

\section{End-weight principle and translation strategy}

Why do above 5 sentences seem familiar? Because we often encounter these sentences in translation and writing, even we ourselves also make these mistakes; the same problem in these 
sentences is that they are top-heavy and imbalanced structurally. Please compare:

7). It is disturbing to think that a dangerous wild animal is still at large in the quiet countryside.

8). Happy are those who are contented.

9). The time has come to explore the South Pole.

10). The story is told of her phenomenal success in Australia.

11) .Decisions have been made to install safety devices on all machines.

Do you feel they are reborn? In fact, we just use some strategies to beautify these sentences, and they can be well used in translation, to greatly enhance the quality of translation. Details are as follows:

Use "it" form subject or form object.

12). It is not uncommon to hear that a shipping company has made a claim for the cost of salvaging a sunken ship.

The longer adverbial is placed at the end of the sentence.

13). He declined the invitation on the grounds of a previous engagement.

Complex direct object is placed at the end of the sentence.

14). He had been known to reduce to tears some of the most seasoned and idolized movie stars in Hollywood.

Complex indirect object is placed at the end of the sentence.

15). He lent some money to each of his five friends.

Avoid too heavy and long subject.

Achieve mainly by dividing subject, inversion, and extraposition and other strategies:

16). No really satisfactory photograph has ever been taken of one in a wild state. (Attributive of one in a wild state and its central word photograph are divided by the predicate)

17). Batteries produce electron flow by chemical means---that is, chemical reactions take place inside the battery that result in a flow of electricity. (Try to compare with: chemical reactions that result in a flow of electricity take place inside the battery.)

18). The day is long gone when oil-company profits transformed the proud new skylines of New Orleans and Houston into testaments to a time of plenty. (The whole attributive clause and its central word ---sentence subject the day --- are divided by the predicate)

19). Einstein concluded that, "In error are those theorists who believe that theory comes inductively from experience". (Try to compare with: Those theorists who believe that theory comes inductively from experience are in error.)

20). There stood one great obstacle---Paris. (Try to compare with: One great obstacle, Paris,stood in the way of this conspiracy.)

\section{Conclusions}

In actual translation, above strategies are very useful, able to solve the English structure balance problem, and help the translator translate sentences with form beauty. In case of conflict between the principle of end-weight and the principle of end-focus, remember that the principle of end-weight obeys the principle of end-focus. Translators must well master and flexibly use them.

\section{References}

[1] Zhao Zhencai. English FAQs Dictionary. Harbin: Heilongjiang People's Publishing House, 1998

[2] Mao Gauoan. Practical English-Chinese Contrast. Chongqing: Chongqing University Press, 2004

[3] Zhang Zhenbang. New English Grammar. Shanghai: Shanghai Foreign Language Education Press, 2003

[4] Chen Wenbo. English-Chinese Translation Techniques and Practice. Beijing: World Knowledge Press, 1998 
[5] Xiong Xueliang, Wang Zhijun. Cognitive Interpretation of Passives. Foreign Language Teaching and Research, 2003 (3)

[6] Chen Wanxia. On Acquisition of English Passive Voice based on Chinese Learner English Corpus. Foreign Language Teaching and Research, 2002 (3)

[7] Leech,G.\& Svartvik .J.A.A Communicative Grammar of English.London:Longmans,1975

[8] In M. Shibatani(ed).Passive and Voice. Amsterdam:John Benjamins. p.329-354

[9] Hallidaym Ak,Hasanr. An introduction to functional grammar .London:EdwardArnold,1988.

[10] Quikr. A grammar of contemporary English.London: Longman,1972. 